\title{
A Deep Learning Algorithms to Generate Activity Sequences Using Historical As-built Schedule Data
}

\author{
Hamed Alikhani ${ }^{1}$, Chau Le $^{2}$ and $\underline{\text { H. David Jeong }}{ }^{3}$
}

1 Interdisciplinary Engineering, Texas A\&M University, College Station, USA, hamedalikhani@tamu.edu

2 Interdisciplinary Engineering, Texas A\&M University, College Station, USA, chle@tamu.edu

3 Department of Construction Science, Texas A\&M University, College Station, USA, djeong@tamu.edu

\begin{abstract}
Project schedule development requires having knowledge about the project's activities and the proper sequence of them. In traditional practice, arranging project activities in a feasible sequential order heavily relies on the project scheduler's practical experience. However, personal experience is limited and prone to include human errors. In this paper, a Deep Learning model is employed to be trained on historical project schedules to predict sequential activities. The proposed model uses a Bidirectional Long Short-Term Memory Recurrent Neural Networks that learns the activity predecessors in the forward direction and the activity successors in the backward direction. The model receives one or more activities and predicts subsequent and precedent activities in a sequential order that have the highest likelihood of occurrence in the historical data. The model is compared with a Sequential Pattern Mining technique that identifies the most probable sequential patterns of activities. The two methods are applied to as-built highway project schedules obtained from a highway agency in the U.S to compare the performance of the two methods. While the Sequential Pattern Mining model provides sequential patterns for certain activities, the Deep Learning model generates a back-tail and a front-tail of activities with any arbitrary length for to provide a more flexible support tool for project schedulers.
\end{abstract}

(c) 2020 The Authors. Published by Budapest University of Technology and Economics \& Diamond Congress Ltd Peer-review under responsibility of the Scientific Committee of the Creative Construction Conference 2020.

Keywords: Deep learning, LSTM, construction scheduling, sequence analysis, highway project schedules

\section{Introduction}

To develop a reliable project schedule, some of the major requirements are the knowledge about the project's activities, required and available resources, production rates, and the sequence logic of the activities. Project activities and quantities are typically identified in the estimated work item amounts in the contract. Production rate estimation of an activity can be can be determined with a reasonable level accuracy when the available resources are identified and historical performance data may provide likely ranges for different activities [1]. However, there are so many possible ways to arrange the order of activities in the project and not all of them result in an efficient plan which makes the activity sequence logic identification a challenging task. Inefficient activity sequence identification results in inaccurate critical path of the project and project duration and contract time determination. Inaccurate estimation of the contract time can lead to an increase in construction cost, inconvenience to the public, and a raise in the number of litigations $[2,3,4]$.

In a typical department of transportation (DOT) in the U.S, schedulers use their own experience to determine the sequence logic of activities for a highway project, and may use guidelines in which a general sequence of activities are introduced [5,6]. However, such guidelines are developed as a high-level guidance based on experts' experience and need frequent updating to reflect the recent requirements of 
standards. Moreover, human experience is limited to a few projects and thus, may cause some bias and is subject to human errors. Research studies tried to address this issue. Typical activity dependency rules have been identified based on physical and material constraints by analysing construction drawing and using experts' knowledge [7, 4]. A recent study developed a data mining model to analyse historical schedules to obtain common sequential patterns among activities [8]. The mining model explored the dataset of historical as-built schedules and identified the most common activity sequential pattern among schedules. Shrestha et al. [8] identified that historical as-built schedules are a valuable resource for identifying the realistic sequence of activities. Although identifying sequential patterns out of historical schedules can provide useful insights for schedulers, there is still a need for a more comprehensive model that can discover sequence insights of the schedules. There is a need that a model not only predict the next successor given one activity, but also the next successors and previous predecessors given one or a short series of activities. This paper developed a Bidirectional Long Short-Term Memory Recurrent Neural Networks (BLSTM) and compare it with a mining technique of Sequential Pattern Mining (SPM) to discuss advantages and disadvantages. The research implemented the two models on 34 real as-built schedule cases of highway project in Montana DOT to validate the results.

\section{Background}

Research studies have been conducted to identify activity dependency rules based on geometry and physical relationship between building components. Cherneff et al. [7] identified rules that determine precedence activities and extracted the geometry, material, and physical constraints information from the project's CAD drawing to help activity sequence identification. The information of geometry, quantity, and physical relationship of project components has also been extracted from BIM models [5]. Some researchers used expert knowledge to identify the common sequence of activities. Jeong et al. [4] obtained Oklahoma DOT schedulers' knowledge to develop fourteen highway scheduling templates. They illustrated activities using flow diagrams to show the sequence, concurrency, and time overlapping of activity implementation in fourteen highway project types. Similarly, Bruce et al. [9] consulted with project schedulers and analyzed historical projects to develop scheduling templates for twelve types of road and bridge projects for Illinois DOT. Montana DOT [6] identified typical sequences of activities for common highway projects. In this study, key controlling activities were described and the time of implementing each activity with regards to other activities was explained.

Some researchers used data-driven methods and analyzed real projects' schedules to obtain common sequential patterns. Shrestha et al. [8] used as-built schedules extracted from Daily Work Report (DWR) data of a State DOT and applied the Sequential Pattern Mining (SPM) technique to identify common activity sequential patterns. For example, they identified that in $78 \%$ of the projects in the dataset, the activity of "cold milling asphalt pavement" was implemented after the "maintenance of traffic". Since the probability of this event is relatively high among all probabilities in the dataset, this sequence pattern was considered as a common sequence. Advanced Artificial Intelligence (Al) tools have also been used for sequence analysis. Long Short-Term Memory (LSTM) [10] is a recurrent neural network architecture which has been applied for sequence analysis such as language models and text prediction to learn the sequence of tasks $[11,12,13]$. Amer and Golparvar-Fard [14] adopted a LSTM model to extract construction activity sequence knowledge using previous construction schedules. They used 12 actual construction project schedules and trained a LSTM model on historical construction schedules that learned the precedence relationships among schedule activities. Given a sequence of activities, the model predicted the next successor activities. Although $\mathrm{Al}$ tools have been adopted to extract sequence knowledge of schedules, there are more challenges ahead; schedulers want to know if they have one or multiple activities, what the most possible predecessors and successors of them are. This paper I) proposes a Deep learning model that is a $\mathrm{Bi}$ directional Long Short-Term Memory (BLSTM), II) applies the model on 34 cases of real overlay project asbuilt schedules to extract sequence knowledge, III) compares the BLSTM results with a most recent activity sequence analysis model of Sequential Pattern Mining (SPM) technique that is applied to the same database to discuss the advantages of the proposed model. 


\section{Data}

Historical as-built schedule data of 34 overlay projects from Montana DOT were used for this study. The data included 42 controlling activities and the projects were constructed is from 2008 to 2016 . The as-built schedule of each project includes the project's controlling activities and the start and end dates of each activity.

\section{Bi-directional LSTM model}

LSTM has been applied in sequence analysis tasks as a sequence to sequence model with an encoder and a decoder. A sequence to sequence model takes a sequence of features as input in the encoder part, and outputs multiple next time steps as a target sequence that follows the input sequence in the decoder part $[15,16]$. The encoder part takes time steps sequence values and passes through a hidden layer to create a final encoding vector that has the information of the input values and the hidden layer. The final encoding vector then is passed to the decoder to generate the next sequential values. If the hidden layer in the encoder part receives and passes the information in one forward direction, the encoder has memorized the information from the past time steps to predict the future, that is called a Unidirectional LSTM. If the hidden layer goes in a forward and a backward direction, the model has learned from the past and future for prediction, which is called a Bidirectional LSTM (BLSTM). Figure 1 shows the architecture of the proposed BLSTM model with an encoder part, input values, final encoding vector, and a decoder part. In the model, $X_{i}$ is the input in the time step $i$ and $Y$ is the output element.

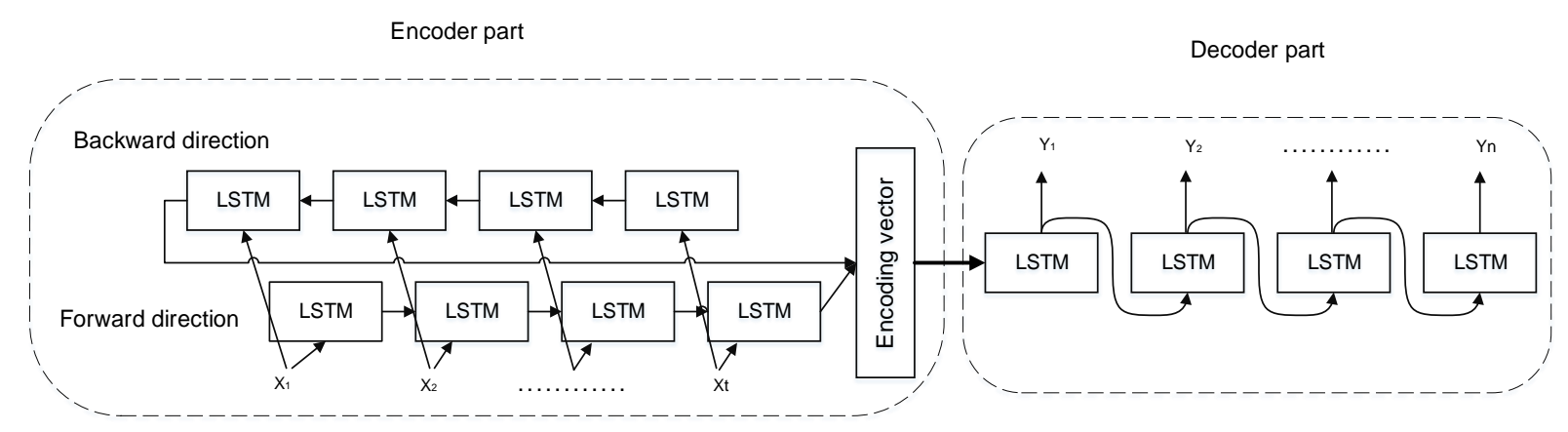

Fig. 1. The architecture of the proposed BLSTM model

The BLSTM model was developed and trained with the as-built schedule database. While training, the model learns the information of previous activities and previous relationships in forward direction and also learns the succeeding relationships via backward direction. The model receives one or many activities and predicts the most common successors and predecessor activities from the database.

Figure 2 illustrates four graphs of sample outputs of the BLSTM model. The model receives one or multiple input activities (green boxes with a bold font) and predicts successors (blue boxes) and predecessors (yellow boxes) with the highest likelihood of occurance in the database. In the first and second graph, one activity is given, and in the third and fourth, two and three sequential activities are given to the model. The numbers show the number of times that the sequence of activities, from the input activities until the specific activities, occurred in the database. For example, in the case \#2, the input activity is "guard rail" that occured 21 times in the database. The model predicts the successors. For example, the sequence of "guard rail- rumble strip" occurred 6 times and the sequence of "guard rail- rumble strip - seal and cover" occurred 3 times and so on. For predecessors, the sequence of "plant mix surfacing - guardrail" occurred 5 times in the database and so on. 


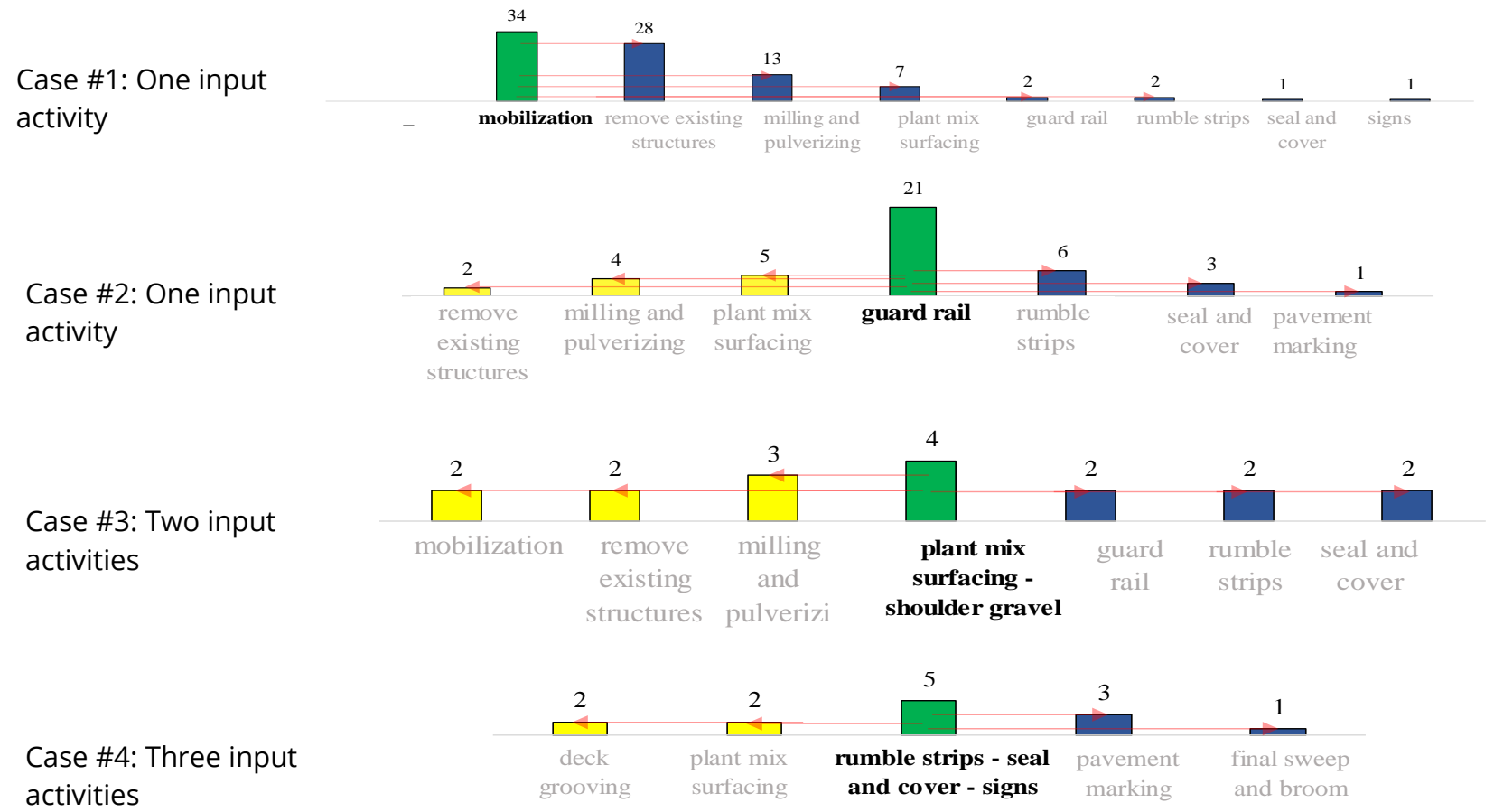

Fig. 1. Four samples of the results of the BLSTM model on the historical as-built schedule data

\section{Sequential pattern mining (SPM) model}

The method of SPM and the application on construction schedules is fully explained in Shrestha et al. [8]. Given a sequence of activities, the SPM discovers all sequential patterns with various lengths. The SPM is applied to the 34 cases of highway schedules and discovered the most common sequential patterns including eight-activities, seven-activities, and other lengths. The results for eight and seven activities are:

- The most common eight-activity pattern: “Mobilization -> Remove Existing Structures -> Milling and Pulverizing -> Plant Mix Surfacing -> Guard Rail -> Rumble Strips -> Seal and Cover -> Pavement Marking".

- The most common seven-activity pattern: "Mobilization -> Remove Existing Structures -> Milling and Pulverizing -> Plant Mix Surfacing -> Guard Rail -> Seal and Cover -> Pavement Marking"

\section{Comparison results}

The results of the BLSTM and SPM models are compared in Table 1. Both models perform the same when it comes to identifying the common patterns. However, the advantage of BLSTM over SPM is the flexibility in receiving one or multiple specific activities and provide arbitrary number of next and previous activities. Although SPM can discover common patterns, it doesn't provide successors of specific activities. The BLSTM can also predict the predecessors as well as successors, because of bidirectional training, while the SPM doesn't predict predecessors. The results of the BLSTM model can be used by schedulers to identify proper activity sequences and relationships. 
Table 1. Comparison of the results of the BLSTM and SPM models

\begin{tabular}{llll}
\hline Task & BLSTM & SPM & \begin{tabular}{l} 
Description \\
\hline $\begin{array}{l}\text { Identifying the most } \\
\text { common } \\
\text { patterns }\end{array}$
\end{tabular} \\
$\begin{array}{l}\text { Predicting successor } \\
\text { activities, given specific } \\
\text { activity/activities }\end{array}$ & $\checkmark$ & Both models perform the same in this task. \\
$\begin{array}{l}\text { Predicting predecessor } \\
\text { activities, given specific } \\
\text { activity/activities }\end{array}$ & $\checkmark$ & $\begin{array}{l}\text { The BLSTM receives spesific activities and predicts the related successors, } \\
\text { while the SPM identifies the common patterns and cannot predict successors for } \\
\text { spesific activities. } \\
\text { The BLSTM can predict successors and predecessors at the same time because } \\
\text { of two-directional training. However, the SPM just work in forward direction and } \\
\text { cannot predict predecessors. }\end{array}$ \\
$\begin{array}{l}\text { Arbitraty number of } \\
\text { inputs and outputs }\end{array}$ & $\times$ & $\begin{array}{l}\text { The BLSTM is flexible in receiving one or multiple activities and predicting } \\
\text { arbitrary number of successors and predecessors. }\end{array}$ \\
\hline
\end{tabular}

\section{Conclusion}

This paper introduced a Deep Learning Bidirectional LSTM (BLSTM) model to learn the sequence of activities in highway projects and trained on real cases of 34 as-built highway project schedules of Montana DOT. The model receives one or a chain of activities and predicts an arbitrary number of predecessor and successor activities. The model can help schedulers to identify the sequence of activities in highway schedules, in that the schedulers give one or multiple activities and the model provides the related activities and their sequential arrangement that are likely to have the highest probability of occurrence in the past projects. The results of the model were compared to a recent sequence analysis model, called SPM algorithm, that was applied on the same dataset. The comparison showed that the BLSTM model is able to predict the next and the past chain of activities at the same time. It is also more flexible in receiving any arbitrary number of input activities and provide the related predecessors and successors.

\section{References}

[1] Woldesenbet, A., D.H.S. Jeong, and G.D. Oberlender, "Daily Work Reports-Based Production Rate Estimation for Highway Projects", Journal of Construction Engineering and Management, 2012 https://doi.org/10.1061/(ASCE)CO.1943-7862.0000442

[2] Federal Highway Administration [FHWA], "FHWA Guide for Construction Contract Time Determination Procedures", 2002, Retrieved from: https://www.fhwa.dot.gov/construction/contracts/t508015.cfm

[3] Hildreth, J. C., "A Review of State DOT Methods for Determining Contract Times", Virginia Tech University, VDOT-VT Partnership for Project Scheduling Charles Edward Via, Jr. Department of Civil and Environmental Engineering, 2005

[4] Jeong, H. S., Atreya, S., Oberlender, G. D., \& Chung, B., "Automated contract time determination system for highway projects", Automation in construction, 2009, 18(7), 957-965 https://doi.org/10.1016/j.autcon.2009.04.004

[5] Kim, H., Anderson, K., Lee, S., \& Hildreth, J., "Generating construction schedules through automatic data extraction using open BIM (building information modeling) technology", Automation in Construction, 2013. 35: p. 285-295. https://doi.org/10.1016/j.autcon.2013.05.020

[6] Montana Department of Transportation (MDT), "Contract Time Determination Procedures", Montana Department of Transportation, Helena, MT, 2008

[7] Cherneff, J., R. Logcher, and D. Sriram, Integrating CAD with Construction- Schedule Generation. Journal of Computing in Civil Engineering, 1991. 5(1): p. 64-84. https://doi.org/10.1061/(ASCE)0887-3801(1991)5:1(64)

[8] Shrestha, K. J., Le, C., Jeong, H. D., \& Le, T, "Mining Daily Work Report Data for Detecting Patterns of Construction Sequences", Creative Construction Conference, Budapest, Hungary, https://doi.org/10.3311/CCC2019-079

[9] Bruce, R.D., et al., An Expert Systems Approach to Highway Construction Scheduling. Technology Interface International Journal, 2012. 13(1): p. 21-28

[10] Hochreiter, Sepp, and Jürgen Schmidhuber. "LSTM can solve hard long time lag problems." Advances in neural information processing systems. 1997.

[11] Spithourakis, Georgios P., Steffen E. Petersen, and Sebastian Riedel. "Clinical text prediction with numerically grounded conditional language models." 2016, arXiv preprint arXiv:1610.06370

[12] Merity, S., Keskar, N. S., \& Socher, R., "Regularizing and optimizing LSTM language models", 2017, arXiv preprint arXiv:1708.02182.

[13] Semeniuta, S., Severyn, A., \& Barth, E., "A hybrid convolutional variational autoencoder for text generation". 2017, arXiv preprint arXiv:1702.02390.

[14] Amer, Fouad, and Mani Golparvar-Fard. "Formalizing Construction Sequencing Knowledge and Mining Company-Specific Best Practices from Past Project Schedules." International Conference on Computing in Civil Engineering, I3CE, June 2019, Atlanta, Georgia. 2019.

[15] Song, L., Zhang, Y., Wang, Z., \& Gildea, D, “A graph-to-sequence model for amr-to-text generation”, 2018, arXiv preprint arXiv:1805.02473.

[16] Battula M., "Time series forecasting with deep stacked unidirectional and bidirectional LSTMs", retrieved from: https://towardsdatascience.com/time-series-forecasting-with-deep-stacked-unidirectional-and-bidirectional-Istms-de7c099bd918, 2019 